\title{
Hepatitis B Vaccination Coverage and Its Associated Fac- tors in Nurses of Imam Reza (AS) Hospital in Kermanshah
}

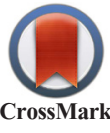

Edris Abdifard ${ }^{1}$, Azadeh Faraji ${ }^{2}$, Elham Sepahvand ${ }^{3}$, Abbas Aghae ${ }^{4}$, Alice Khachian ${ }^{5 *}$

1. Students Research Committee, Kermanshah University of Medical Sciences, Kermanshah, Iran.

2. Department of Nursing, School of Nursing \& Midwifery, Iran University of Medical Sciences, Tehran, Iran.

3. Department of Nursing, University of Social Welfare and Rehabilitation Sciences, Tehran, Iran.

4. Department of Statistics and Epidemiology, School of Health, Shahid Beheshti University of Medical Sciences, Tehran, Iran.

5. Department of Medical Surgical Nursing, School of Nursing \& Midwifery, Iran University of Medical Sciences, Tehran, Iran.

citation:Abdifard, E, Faraji, A, Sepahvand, E, Aghae, A \& Khachian, A 2016, 'Hepatitis B Vaccination Coverage and Its Associated Factors in Nurses of Imam Reza (AS) Hospital in Kermanshah', Journal of Client-Centered Nursing Care, vol. 2, no. 2, pp. 97-102. https://doi.org/10.32598/jccnc.2.2.97

: https://doi.org/10.32598/jccnc.2.2.97

Article info:

Received: 10 Nov. 2015

Accepted: 13 Feb. 2016

Keywords:

Vaccination, Hepatitis B,

Nurses, Iran

\section{A B S T RA C T}

Background: Viral hepatitis is one the main infections that health staff are faced with and nurses are at greater risk of the contracting this disease because of their exposure to patients. The current study was conducted to determine the hepatitis B vaccination coverage and its associated factors in nurses of Imam Reza (AS) Hospital in Kermanshah, Iran.

Methods: In this descriptive-analytic study, 258 nurses working in Imam Reza (AS) Hospital of Kermanshah were studied based on census records in 2012. Instrument for the data collection was a researcher-made questionnaire, which its validity and reliability were approved. Collected data were analyzed by the Chi-square and ANOVA tests using SPSS 16.

Results: Out of 258 nursing staff, $85.2 \%$ had completed vaccination, $3.9 \%$ had it once, and $10.9 \%$ had received two shots of vaccine. Among the most important barriers to vaccination mentioned by the participants were forgetting $(57.2 \%)$, negligence $(39.5 \%)$, and high workload $(36.8 \%)$

Conclusion: Given the relatively high coverage of vaccination in this study, to maintain the efficiency of nurses as well as reducing the cost of difficult treatment of hepatitis B, necessary facilities and education about the disease and its complications should be provided by the authorities for the full vaccination coverage at very low cost.

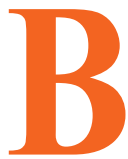

\section{Introduction}

ecause of its many risk factors and different ways of spread, Hepatitis B in- fection is seen in all ages and all around the world. According to global statistics, more than 2 billion people are infected with the hepatitis B virus, around 350 million $(5 \%)$ of them are chronic virus carriers.

* Corresponding Author:

Alice Khachian, PhD

Address: Department of Medical Surgical Nursing, School of Nursing \& Midwifery, Iran University of Medical Sciences, Tehran, Iran.

Tel: +98 (21) 43651708

E-mail: khachian.a@iums.ac.ir 
Every year more than 1 million people infected with hepatitis B virus die as a result of its complications such as cirrhosis or cancer of the liver (Amini-Ranjbar \& Motlagh 2008; Civljak et al. 2013). According to the World Health Organization and the Center for Disease Control and Prevention, Iran ranks among countries with average endemicity of hepatitis B (Poorolajal \& Majdzadeh 2009).

Different ways of transmission of hepatitis include blood transfusion, body fluids, mother to child, and occupational injuries (Rabiei et al. 2008). One of the occupational hazards for health staff who are in contact with human blood secretions, is infection with hepatitis B virus (Civljak et al. 2013). Unavoidable contact with patients and the use of medical equipment increase the risk of infection among the treatment team personnel. Around the world, the prevalence of hepatitis B virus infection among the medical staff is $5.9 \%$ (Attaullah et al. 2011). Although the prevalence of hepatitis B virus among health staff is different among countries, in developing countries, the prevalence of hepatitis B virus infection is between $40 \%-60 \%$ among treatment team staff and in developed countries it is less than 10\% (Attaullah et al. 2011). The risk of infection as a result of once-contact is between $6 \%-30 \%$. This virus can resist for months in the external environment under suitable conditions and this factor can justify why hepatitis B is an occupational hazard for health staff (Tsoulas \& Apostolopoulou 2009).

Among the healthcare staff, nurses are at greater risk due to their frequent contacts with the blood or secretions of the patients (Ganczak et al. 2010). In a study by Abdi et al. in 2013 in Kermanshah, it was observed that $73.3 \%$ of the studied nurses had experienced needle stick injury and $64 \%$ of these injuries had occurred in the past year (Abdifard et al. 2015). It is clear that prevalence of hepatitis B infection can be reduced by general and specific prophylactic measures, including donor screening, use of disposable medical equipment, sterilization of reusable devices, providing educational programs for chronic virus vectors and vaccination to reduce transmission of the virus (Toska et al. 2011). Hepatitis B is a disease preventable by vaccination. In other words, the most effective strategy to prevent transmission of the hepatitis B virus is vaccination. Hepatitis B vaccine is one of the most widely used vaccines around the world (Ganczak et al. 2010). The purpose of the immunization program against hepatitis $\mathrm{B}$ is prevention of disease infection, becoming a vector of the virus and in other words, elimination of chronic vectors of hepatitis B (Topuridze et al. 2010).
In the study by Salari et al. conducted on healthcare workers in 2006 , about $68 \%$ of them completed vaccination coverage, $26.4 \%$ had incomplete coverage and $5.6 \%$ of subjects had not been vaccinated (Salary et al. 2007). In the study by Ganczak in 2009 on gynecologic and surgery nurses, $100 \%$ of nurses had complete vaccination coverage, although only for $21 \%$ of personnel had been checked for hepatitis antibodies (Ganczak et al. 2010). Given the importance of vaccination of the health staff, a new report on vaccination coverage, its rate, and assessing the level of health protection among staff can provide insight into vaccination status and the development of a proper vaccination program. This study was conducted in 2012 with the aim of assessing vaccination coverage rates and related factors among nurses of Imam Reza (AS) Hospital in Kermanshah, Iran.

\section{Materials and Methods}

This cross-sectional study was conducted on nurses working in Imam Reza (AS) Hospital of Kermanshah affiliated to Kermanshah University of Medical Sciences. By referring to all medical units of Imam Reza (AS) Hospital, researchers selected and enrolled 258 nurses for this study. Data collection instrument was a researcher-made questionnaire which was prepared in three parts. The first part had 9 questions asking about demographic information, second part contained 11 questions regarding employment status of subjects and the third part consisted of 7 questions about the history of vaccination for hepatitis $\mathrm{B}$, the number of vaccinations, and factors related to failure to perform or complete vaccination (including lack of awareness, forgetting, high workload, negligence, disease at periodic intervals vaccination, low risk of injury with sharp objects, non-occupational exposure to patients with hepatitis B, lack of belief in the efficacy of vaccination, dangerous vaccine, impatience, the unavailability of vaccines, medical ban, and sensitivity to vaccine against hepatitis B).

Validity of the study was confirmed by content validity, a review of the literature and verification by the experts. Its reliability was also confirmed by the testretest method after completing the questionnaire by 16 nurses (79.4\%). Inclusion criterion of the study was employment in Imam Reza (AS) Hospital and exclusion criteria were having diagnosed with $\mathrm{HBV}, \mathrm{HCV}$, or HIV, serious systemic and metabolic disorders, and evidence of cancers in participants, and also filling out the questionnaires incompletely. Collected data were fed into SPSS version 16. All information was analyzed by calculating mean, standard deviation, and 
frequency of qualitative and quantitative variables using the Chi-square test and analysis of variance (at a significance level of $\mathrm{P} \leq 0.05$ ).

\section{Results}

A total of 258 questionnaires were completed and analyzed. Of participants, 202(78.3\%) were females and $56(21.7 \%)$ were males. The Mean \pm SD age of studied nurses was $32.35 \pm 6.1$ years, the Mean $\pm \mathrm{SD}$ duration of clinical work s w8.48 \pm 5.88 years and Mean $\pm \mathrm{SD}$ of overtime work of nurses was $82.48 \pm 47$ hours per month. Majority of them were married (58.9\%), had a BA degree $(93.4 \%)$, and in terms of shifts, had rotating shifts (78.3\%), and were working in intensive care units $(34.1 \%)$. The results also showed that most subjects under study $(85.7 \%)$ had a history of caring patients with hepatitis B (HBSAg positive). About 8.5\% of subjects had personal history of hepatitis B (HBSAg positive) resulting from occupational exposure. The majority of subjects $(73.3 \%)$ mentioned history of injuries with sharp instruments in the work place. About $28.4 \%$ of subjects mentioned history of contact with infected fluids and secretions of the patients with hepatitis B. The majority of subjects $(86.4 \%)$ have used protective equipment when performing invasive procedures and patient care (gloves, masks, and goggles, if necessary). More than half of the subjects (57\%) have encountered with the cases where protective equipment was not available to them when performing invasive procedures and caring patients.

About $99.2 \%$ reported history of vaccination, $85.2 \%$ completed vaccination, $3.9 \%$ of them had a single shot of vaccination, and $10.9 \%$ received two shots of vaccination. In this study, only half of nurses (51.4\%) had measured antibody titers a month after the third dose of vaccine received. Also nurses in this study have mentioned most frequent reasons of incompletion of vaccination as forgetting $(57.2 \%)$, negligence (39.5\%), and high workload (36.8\%).

Table 1 showed that highest rate of coverage was $87.3 \%$ among married people, $86.1 \%$ in women, $92.9 \%$ in Deputy Head nurses, $94.7 \%$ in emergency department and operating room nurses, and $93.3 \%$ in those with MS degree.

The above Table shows that the highest vaccination coverage was $87.3 \%$ among married couples, $86.1 \%$ in women, $92.9 \%$ in Deputy Head nurses, $94.7 \%$ in emergency department and operation room nurses, and $93.3 \%$ in those with a master's degree. Also, with regard to the relationship of partial or complete vac-

Table 1. Assessing the vaccination coverage with regard to demographic variables of nurses in Imam Reza (AS) Hospital in 2012.

\begin{tabular}{|c|c|c|c|c|}
\hline Study participants & & $\begin{array}{l}\text { Partial vaccination } \\
\text { (one or two shots) }\end{array}$ & $\begin{array}{c}\text { Complete vaccination } \\
\text { (three shots) }\end{array}$ & P-value \\
\hline Mean(SD) age, y & & $34.32(0.6)$ & $31.92(5.93)$ & $P=0.008$ \\
\hline $\begin{array}{l}\text { Mean(SD) history of clinical } \\
\text { work, y }\end{array}$ & & $10.47(6.53)$ & $8.01(5.62)$ & $P=0.007$ \\
\hline \multirow{2}{*}{ Gender } & Female & $13.9 \%$ & $86.1 \%$ & \multirow{2}{*}{$P=0.403$} \\
\hline & Male & $18.2 \%$ & $81.8 \%$ & \\
\hline \multirow{3}{*}{ Position } & Head nurse & $15.4 \%$ & $84.6 \%$ & \multirow{3}{*}{$P=0.672$} \\
\hline & Deputy head nurse & $7.1 \%$ & $92.9 \%$ & \\
\hline & Nurse & $15.3 \%$ & $84.7 \%$ & \\
\hline \multirow{4}{*}{ Section of workplace } & Internal & $16.9 \%$ & $83.1 \%$ & \multirow{4}{*}{$P=0.139$} \\
\hline & Operation & $22.2 \%$ & $77.8 \%$ & \\
\hline & $\mathrm{CCU}$ & $12.6 \%$ & $87.4 \%$ & \\
\hline & $\begin{array}{l}\text { Emergency and } \\
\text { operation room }\end{array}$ & $5.3 \%$ & $94.7 \%$ & \\
\hline \multirow{3}{*}{ Education degree } & Associate degree & $50 \%$ & $50 \%$ & \multirow{3}{*}{$P=0.256$} \\
\hline & Bachelor's degree & $15.1 \%$ & $84.9 \%$ & \\
\hline & Master's degree & $6.7 \%$ & $93.3 \%$ & \\
\hline \multirow{2}{*}{ Marital status } & Single & $17.9 \%$ & $82.1 \%$ & \multirow{2}{*}{$P=0.387$} \\
\hline & Married & $12.7 \%$ & $87.3 \%$ & \\
\hline
\end{tabular}


cination course with the demographic characteristics, a significant relationship was seen only between the lack or incomplete completion of vaccination courses with age $(\mathrm{P}=0.008)$ and clinical experience $(\mathrm{P}=0.007)$.

\section{Discussion}

Based on the results of this study, $85.2 \%$ (218 persons) had completed their vaccination that this is more than results from internal and external studies such as Amini Ranjbar and Ismail Motlagh in 2008 in Kerman teaching hospitals (71.7\%) and Attaullah et al. in 2011 in Pakistan nurses (65.4\%) (Izadpanah et al. 2014). In Gholami study, also vaccination coverage rates was at favorable level among nurses (Gholami et al. 2010). In a study in 2011 in South Africa, 67.6\% of nurses had mentioned history of completed vaccination (Burnett et al. 2011). In a study by Pathak et al. (2013), in India, only $44.8 \%$ of nurses reported complete, $9 \%$ incomplete vaccination coverage, and $45.6 \%$ reported no vaccination (Pathak et al. 2013) which are far less than this study results.

The high rate vaccination of the married subjects in this study is not consistent with the result of Salary et al. (2006) in which the coverage rate $(82.9 \%)$ in single people was significantly higher than the married (5.66\%) subjects (Salary et al. 2007). In justifying this, it can be argued that married people had paid more attention to hepatitis $\mathrm{B}$ vaccines because of being aware of hepatitis B transmission through sexual contact which increases the risk of infection by the disease in married subjects.

Significant differences were also observed in a study by Salari et al. regarding the age in the participants with complete coverage of vaccination and those with incomplete coverage and those with no coverage at all $(\mathrm{P}=0.008)$ (Table 1) (Salary et al. 2007). But in the study by Izadpanah et al. (2002), no statistically significant difference was observed (Izadpanah et al. 2014). Also, there is a significant difference regarding clinical experience in nurses with complete coverage and those with incomplete coverage and those with no coverage in this study $(\mathrm{P}=0.007)$ (Table 1$)$. The results were consistent with the results of Attaullah et al. (2011) study conducted on the hospital staff in Pakistan (Attaullah et al. 2011). Significant correlation between vaccination coverage and the mean age and mean duration of the clinical history may reflect the fact that young and new graduates have paid more attention to prophylaxis and receiving vaccinations, because of having more information about hepatitis B and its complications.
In terms of educational level, $50 \%$ of coverage rate belonged to those with an associate degree, $84.9 \%$ to those with bachelor's degree, and $93.3 \%$ to those with master's degree. The difference observed was not statistically significant (Table 1) and this finding was not consistent with the Izadpanah study results (Izadpanah et al. 2014).

Today in all hospitals, awareness of the prophylaxis and prevention of occupational diseases matter for all health care staff regardless of their degree and position. Given that only $51.4 \%$ of nurses in this study had measured antibody titers a month after the third dose of vaccination, it is suggested that after one to three months, antibody test against hepatitis B be performed for all nurses, and relevant actions be taken for the revaccination or booster injection as needed. Furthermore, causes for negative response and confounding factors are investigated by identifying people who have shown a negative response.

In this study, most nurses attributed reasons of incompletion of vaccination course to forgetting $(57.2 \%)$, negligence (39.5\%), and high workload (36.8\%). In a study in Peshawar, Pakistan (2011), most reasons to vaccination uncoverage reported to be due to high workload $(39.8 \%)$, negligence $(38.8 \%)$, problem in the preparation of the vaccine $(20.9 \%)$, and the unavailability of vaccine (0.5\%) (Attaullah et al. 2011) In another study conducted by Amini-Ranjbar and Ismail Motlagh (2008) on the nursing staff of teaching hospitals in Kerman, negligence (44.6\%), the unavailability of the vaccine $(27.7 \%)$, fear of the vaccine or its complications $(9.2 \%)$, and lack of awareness (6.2\%) were reported as the most important barriers to vaccination (Amini-Ranjbar \& Motlagh 2008). Negligence $(54.4 \%)$, lack of awareness of the need for vaccination $(35 \%)$, and high cost of vaccination $(17 \%)$ were among the most important barriers to vaccination in Tehran physicians (Alavian \& Hatami 2003).

Providing necessary facilities and vaccination requirements for hospitals and increasing the level of knowledge about the importance of vaccination and completing booster doses can overcome these barriers.

Hepatitis B vaccination coverage was $85.2 \%$ among the nurses of healthcare team. Lack of knowledge, attitude, and performance are the main reasons for the lack of complete vaccination coverage among nurses. Learning more about hepatitis B and vaccination against it can correct some wrong beliefs and myths and promote public health. Also training nursing personnel regarding the use of protective equipment for 
the disease has been effective and can be adopted as a training protocol in all hospitals. Learning programs should be focused on receiving vaccination shots during occupational exposure to hepatitis B and an emphasis on safety and effectiveness of vaccines. An increase in the availability of vaccination may be another effective method for increase in acceptance of hepatitis B vaccination. Therefore, national policies for mandatory vaccination against hepatitis B must be implemented.

\section{Acknowledgments}

The current study was approved (Project No. 91050) by Student Research Committee of Kermanshah University of Medical Sciences which was carried out in 2012-2013. The researchers hereby express their gratitude for financial support from the University Vice Chancellor for Research and Technology in this project. Finally, we hoped that results of this study be useful for respected authorities. Also, we appreciate the assistance of respected authorities of Imam Reza (AS) Hospital in Kermanshah.

\section{Conflict of Interest}

The authors declared no conflict of interest.

\section{References}

Abdifard, E, Sepahvand, E, Aghaei, A, Hosseini, S \& Khachian, A 2015, 'Needle-stick, sharp injuries and its related factors among nurses of Imam Reza Hospital, Kermanshah, 2013, Journal of Client-Centered Nursing Care, vol. 1, no. 4, pp. 183-88.

Alavian, SM \& Hatami, S 2003, '[Hepatitis B vaccination rates among physicians practicing in Tehran (Persian)]', Medical Council Journal, vol. 21, no. 3, pp. 204-207.

Amini-Ranjbar, S \& Motlagh, ME 2008, 'Hepatitis B vaccination coverage among Iranian medical students and nursing staff', American Journal of Applied Sciences, vol. 5, no. 6, pp. 747-749. doi: 10.3844 /ajassp.2008.747.749

Attaullah, S, Khan, S \& Ayaz S 2011, 'Prevalence of HBV and $\mathrm{HBV}$ vaccination coverage in health care workers of tertiary hospitals of Peshawar, Pakistan. Virology Journal, no. 16, p. 275. doi: $10.1186 / 1743-422 X-8-275$

Burnett, RJ, François, G, Mphahlele, MJ, Mureithi, JG, Africa, PN, Satekge, MM et al. 2011, 'Hepatitis B vaccination coverage in healthcare workers in Gauteng Province, South Africa. Vaccine, vol. 29, no. 25, pp. 4293-7. doi: 10.1016/j.vaccine.2011.03.001

Civljak, R, Papic, N, Stamenic, V, Kalenic, S, Kuzman, I \& Car, J 2013, 'Influenza and hepatitis B vaccination coverage among healthcare workers in Croatian hospitals: A series of crosssectional surveys, 2006-2011', BMC Infectious Diseases, no. 13 p. 520. doi: 10.1186/1471-2334-13-520

Ganczak, M, Ostrowski, M, Szych, Z, Korzeń, M 2010, 'A complete $\mathrm{HBV}$ vaccination coverage among Polish surgical nurses in the light of anti-HBc prevalence: A cross-sectional seroprevalence study. Vaccine, vol. 28, no. 23, pp. 3972-976. doi: 10.1016/j.vaccine.2010.03.042

Gholami, A, Alinia, T \& Moosavi Jahromi, L 2010, '[Hepatitis B vaccination coverage among teaching hospitals workers in the Urmia city (Persian)]', Urmia University of Medical Sciences, vol. 8, no. 4, pp. 212-19.

Izadpanah, AM, Mashreghi moghadam, HR \& Mogharab, M 2004, '[Study of Hepatitis B vaccination coverage among nursing staff Birjand University of Medical Sciences (Persian)]', Journal of Birjand University of Medical Sciences, vol. 11, no. 4, pp. $49-46$

Pathak, R, Chaudhary, CH, Pathania, D, Ahluwalia, SK, Mishra, PK \& Kahlon, AS 2013, 'Hepatitis B vaccine: Coverage and factors relating to its acceptance among health care workers of a tertiary care center in North India', International Journal of Medicine and Public Health, vol. 3, no. 1, pp. 55-9. doi: 10.4103/22308598.109324

Poorolajal, J \& Majdzadeh, R 2009, '[Prevalence of chronic hepatitis B infection in Iran: a review study (Persian)]', Iranian Journal of Epidemiology, vol. 4, no. 3, pp.1-8.

Rabiei, M, Mohtasham Amiri, Z \& Masoudi Rad, H 2008, '[Survey of $\mathrm{HBV}$ vaccination among dentists and their staffs in Guilan (Persian)]', Journal of Guilan University of Medical Sciences, vol. 64 , no. 16 , pp. 37-42.

Salary, MM, Alavian, SM \& Tadrisi, SD 2007, '[Evaluation of vaccination coverage and immunity to hepatitis B among healthcare workers (Persian)]', Kowsar Medical Journal, vol. 11, no. 4, pp. 343-52.

Topuridze, M, Butsashvili, M, Kamkamidze, G, Kajaia, M Morse, D \& McNutt, LA 2010, 'Barriers to Hepatitis B vaccine coverage among healthcare workers in the Republic of Georgia: an international perspective', Infection Control \& Hospital Epidemiology, vol. 31, no. 2, pp. 158-64. doi: 10.1086/649795

Toska, AG, Saridi, M, Giolis, A, Kyriazis, I, Pappa, V, Androulakis, E et al. 2011, 'Hepatitis B vaccination coverage levels among nurses in Greece: need for improvement', Southern Medical Journal, vol. 39, no. 6, pp. 74-76. doi: 10.1097/ smj.0b013e31820bfcce

Tsoulasm, D \& Apostolopoulou, E 2009, 'Hepatitis B vaccination coverage levels among health care workers in Greek Military Hospitals', Balkan Military Medical Review, vol. 12, no. 4, pp. $133-42$. 
